\title{
Cholesterol levels and long-term rates of community-acquired sepsis
}

\author{
Faheem W. Guirgis ${ }^{1}$, John P. Donnelly 2,3 , Sunita Dodani ${ }^{6,7}$, George Howard ${ }^{8}$, Monika M. Safford ${ }^{4,5}$, \\ Emily B. Levitan ${ }^{3}$ and Henry E. Wang ${ }^{2,9^{*}}$
}

\begin{abstract}
Background: Dyslipidemia is a risk factor for cardiovascular disease, with elevated low-density lipoprotein cholesterol $(\mathrm{LDL}-\mathrm{C})$ and decreased high-density lipoprotein cholesterol (HDL-C) recognized as risk factors for acute coronary events. Studies suggest an association between low cholesterol levels and poor outcomes in acute sepsis. We sought to determine the relationship between baseline cholesterol levels and long-term rates of sepsis.

Methods: We used data from the Reasons for Geographic and Racial Differences in Stroke (REGARDS) cohort, a population-based cohort of 30,239 community-dwelling adults. The primary outcome was first sepsis event, defined as hospitalization for an infection with the presence of $\geq 2$ systemic inflammatory response syndrome criteria (abnormal temperature, heart rate, respiratory rate, white blood cell count) during the first 28 hours of hospitalization. Cox models assessed the association between quartiles of HDL-C or LDL-C and first sepsis event, adjusted for participant demographics, health behaviors, chronic medical conditions, and biomarkers.
\end{abstract}

Results: We included 29,690 subjects with available baseline HDL-C and LDL-C. There were 3423 hospitalizations for serious infections, with 1845 total sepsis events among 1526 individuals. Serum HDL-C quartile was not associated with long-term rates of sepsis (hazard ratio (HR) (95\% Cl): Q1 (HDL-C 5-40 mg/dl), 1.08 (0.91-1.28); Q2 (HDL-C 41-49 mg/dl), 1.06 (0.90-1.26); Q3 (HDL-C 50-61 mg/dl), 1.04 (0.89-1.23); Q4, reference). However, compared with the highest quartile of LDL-C, low LDL-C was associated with higher rates of sepsis (Q1 (LDL-C 3-89 mg/dl), 1.30 (1.10-1.52); Q2 (LDL-C 90-111 mg/dl), 1.24 (1.06-1.47); Q3 (LDL-C 112-135 mg/dl), 1.07 (0.91-1.26); Q4, reference).

Conclusion: Low LDL-C was associated with higher long-terms rates of community-acquired sepsis. HDL-C level was not associated with long-term sepsis rates.

Keywords: Lipids, Prevention, Infection, Inflammation

\section{Background}

One of the greatest accomplishments for preventive medicine has been the recognition and treatment of hyperlipidemia to prevent coronary artery disease (CAD). At the forefront has been the institution of HMG-CoA reductase inhibitor (statin) drug therapy to lower low-density lipoprotein cholesterol (LDL-C) to target levels with significant benefit [1]. More controversial, however, has been the association between lower levels of high-density lipoprotein cholesterol (HDL-C) and increased risk of

\footnotetext{
*Correspondence: Hwang@uabmc.edu

${ }^{2}$ Department of Emergency Medicine, University of Alabama School of

Medicine, Birmingham, AL, USA

${ }^{9}$ Department of Emergency Medicine, University of Alabama at Birmingham,

619 19th Street South, OHB 251, Birmingham, AL 35249, USA

Full list of author information is available at the end of the article
}

CAD. Atherosclerosis occurs when LDL-C particles become trapped in the subendothelial space of human artery cell walls and attract inflammatory cells. Generally, HDL-C is protective because it transfers LDL-C to the liver and adrenal glands and facilitates its elimination in the intestinal tract. HDL-C also has several antioxidant and anti-inflammatory properties which can help prevent LDL-C oxidation and inflammatory cell migration. The landmark Framingham study demonstrated that low HDL-C was a risk factor for CAD death in both males and females [2]. However, recent studies found no additional benefit to raising serum HDL-C levels with medications in patients with optimized LDL-C already on statins [3]. 
In addition to $\mathrm{CAD}$, manipulation of cholesterol levels may also play an important role in combating sepsis, the syndrome of dysregulated response to microbial infection. Sepsis results in approximately 570,000 annual emergency department (ED) visits in the United States [4]. Nearly 215,000 patients die from sepsis each year [5] and sepsis has an estimated cost of $\$ 16.7$ billion annually in the United States alone [6]. The most compelling evidence for the role of lipids in protection against sepsis involves HDL-C. There are several mechanisms by which HDL-C has been shown to be protective in sepsis, including bacterial toxin binding and disposal $[7,8]$, monocyte suppression, macrophage and dendritic cell migration, release of inflammatory cytokines $[9,10]$, and inhibition of vascular and intercellular adhesion molecule expression [11]. All of these processes can lead to dysregulated inflammation and endothelial injury, which can result in the clinical manifestations of organ damage and death. Low HDL-C levels have also been associated with poor sepsis outcomes [12-14]. In addition, LDL-C has been shown to facilitate bacterial toxin clearance in sepsis [15]. Lagrost et al. [16] demonstrated that lower baseline total cholesterol levels were present in patients who developed sepsis after cardiac surgery.

In prior studies, we have demonstrated that cardiovascular disease is a risk factor for incident sepsis events [17]. The association between baseline cholesterol and rates of sepsis remains unknown. As with $\mathrm{CAD}$, a relationship between cholesterol and sepsis could provide an opportunity for potentially reducing an individual's longterm risk through targeting of particular lipids. In this study, we sought to determine the association between baseline lipid levels and long-term rates of sepsis among community-dwelling adults in the Reasons for Geographic and Racial Disparities in Stroke (REGARDS) cohort.

\section{Methods}

\section{Study design}

We performed a prospective cohort study using data from the REGARDS study, a national population-based longitudinal cohort.

\section{Data source}

REGARDS is a 30,239-participant cohort study of community-dwelling adults $\geq 45$ years of age. The cohort recruited participants from 2003 to 2007 [18]. The cohort is $45 \%$ male, $41 \%$ African American, and 69\% aged $>60$ years. The study oversampled Black individuals and those living in the southeastern United States, with $21 \%$ of the cohort originating from the coastal plains of North Carolina, South Carolina, and Georgia (the "stroke buckle"), and 35\% from the remainder of North Carolina, South Carolina, and Georgia plus Tennessee,
Mississippi, Alabama, Louisiana, and Arkansas (the "stroke belt").

Baseline data obtained upon participant enrollment included sociodemographics, health behaviors, chronic medical conditions, as well as information on diet, family history, psychosocial factors, and prior residences. In addition, blood and urine specimens were obtained from each participant. The study contacts participants every 6 months to determine the date, location, and reason for all hospitalizations.

\section{Identification of sepsis events}

We identified all hospitalizations attributed by participants to a serious infection; for example, pneumonia, pyelonephritis, urinary tract infection, diverticulitis, meningitis, and cellulitis, among others. Two trained research assistants independently conducted a structured review of all retrieved medical records, including ED physician and nursing, admission, progress and discharge notes, laboratory reports, vital sign tables, and radiology reports. We reviewed records for the first 28 hours of hospitalization to allow for ED treatment plus one full day of hospitalization. Sepsis events were defined as a hospitalization for an infection with the presence of $\geq 2$ systemic inflammatory response syndrome (SIRS) criteria (temperature $<36$ or $>38{ }^{\circ} \mathrm{C}$, heart rate $>90$ beats per minute, respiratory rate $>20$ breaths per minute or $\mathrm{PaCO}_{2}<32 \mathrm{mmHg}$, white blood cell count $<4000$ or $>12,000 \mathrm{cell} / \mathrm{mm}^{3}$ ) during the first 28 hours of hospitalization. We did not consider sepsis events occurring at later points of hospitalization. We assessed sepsis events occurring over a 10-year time period from January 1, 2003 through December 31, 2012.

\section{Participant and hospitalization characteristics}

Participant characteristics were determined during the initial interview and in-home visit. Demographics included age, race, sex, income, education, and geographic location. Race was defined as White or Black/African American, and was self-reported. Income was divided into four categories $(<\$ 20,000, \$ 20,000-34,000, \quad \$ 35,000$ $74,000, \geq \$ 75,000)$. Education categories included less than high school, high school graduate, some college, and college or higher. Geographic region included the "stroke buckle" (coastal plains of North Carolina, South Carolina, and Georgia), the "stroke belt" (remainder of North Carolina, South Carolina, and Georgia plus Tennessee, Mississippi, Alabama, Louisiana, and Arkansas), and nonbelt. Health behaviors included cigarette smoking (current, past, never) and alcohol use (moderate use (1 drink per day for women or 2 drinks per day for men), heavy use ( $>1$ drink per day for women and $>2$ drinks per day for men) as per the National Institute on Alcohol Abuse and Alcoholism classification). 
Chronic medical conditions included cancer, CAD, diabetes, hypertension, obesity, and stroke. Participants self-reported history of cancer, myocardial infarction, or stroke. CAD included a history of myocardial infarction or electrocardiogram evidence of prior MI [19]. Diabetes was defined as fasting glucose $\geq 126 \mathrm{mg} / \mathrm{L}$ (or glucose $\geq 200 \mathrm{mg} / \mathrm{L}$ for those not fasting) or the use of insulin or oral hypoglycemic agents. Hypertension included systolic blood pressure $\geq 140 \mathrm{mmHg}$, diastolic blood pressure $\geq 90 \mathrm{mmHg}$, or the reported use of antihypertensive agents. Obesity included a body mass index $\geq 30 \mathrm{~kg} / \mathrm{m}^{2}$ or waist circumference $>102 \mathrm{~cm}$ for males or $>88 \mathrm{~cm}$ for females.

REGARDS did not collect information on pulmonary conditions such as asthma and chronic obstructive pulmonary disease. Therefore, we defined participant use of pulmonary medications as a surrogate for chronic lung disease. Obtained from each participant's medication inventory, pulmonary medications included beta- 2 adrenergic agonists, leukotriene inhibitors, inhaled corticosteroids, combination inhalers, and other pulmonary medications such as ipratropium, cromolyn, aminophylline, and theophylline. Statin use was defined in a similar manner, with specific statins reported by subjects including atorvastatin, fluvastatin, lovastatin, pravastatin, rosuvastatin, and simvastatin. In order to account for medication adherence, we also obtained participant responses to the four-question version of the Morisky Medication Adherence Scale, which provides a measure of individual compliance with medication use (scale ranges from 0 to 4 , with 0 indicating good adherence and 4 indicating poor adherence) [20].

We used data on biomarkers obtained for all subjects, including high-sensitivity C-reactive protein (hs-CRP), cystatin $\mathrm{C}$, urinary albumin-to-creatinine ratio (ACR), and estimated glomerular filtration rate (eGFR). Study personnel collected blood and urine samples from all REGARDS participants at subjects' homes following a 10-12-hour fast. Samples were centrifuged to separate serum or plasma within 2 hours of collection and shipped overnight on ice packs to the laboratories at the University of Vermont. On arrival, study personnel centrifuged the samples at $30,000 \times g$ and $4{ }^{\circ} \mathrm{C}$. The samples were either analyzed (general chemistries) immediately or stored at $-80{ }^{\circ} \mathrm{C}$.

Functional status measures included the 12-Item Short Form Health Survey (SF-12) physical composite score (PCS) in addition to self-reported physical activity and exhaustion. Consistent with established definitions, we defined physical weakness as $\mathrm{PCS}<75$, low weekly physical activity as responses of "almost never" or "never" to the question "How many times per week do you engage in intense physical activity, enough to work up a sweat?" and exhaustion as responses of "a little of the time" or "none of the time" to the question "How much of the time during the past 4 weeks did you have energy?" [21-23].

Among participants hospitalized with sepsis, we identified several hospitalization characteristics via chart review of available clinical data. We identified sepsis events with elevated Sequential (sepsis-related) Organ Failure Assessment (SOFA) score ( $\geq 2$ points) using established criteria $[24,25]$. We also identified admission destination and inhospital mortality.

\section{Primary exposure}

Serum total cholesterol, HDL-C, and triglyceride levels were directly measured from serum samples. LDL-C was calculated using the Friedewald formula from total cholesterol, HDL-C, and triglyceride [26]. Our primary exposures were HDL-C and LDL-C quartiles. We also classified lipid levels using established clinical cutoff values for HDL-C $(<40,40-59$, and $\geq 60 \mathrm{mg} / \mathrm{dl})$ and LDL-C $(<130,130-159$, and $\geq 160 \mathrm{mg} / \mathrm{dl})$ [1]. We also analyzed lipid values as continuous variables and as percentages of total cholesterol (per standard deviation increase).

\section{Data analysis}

We compared baseline participant characteristics across HDL-C and LDL-C quartiles using Pearson chi-square tests of association and $t$ tests of equal means for categorical and continuous variables, respectively. Because we observed visual trends in biomarker levels based on lipid level quartiles, we specified tests for linear trend using modified Poisson regression for a common outcome, including abnormal biomarker as the dependent variable with HDL-C or LDL-C quartile as the independent variable.

In order to determine the association between LDL-C and HDL-C and rates of sepsis, we fit a series of Cox proportional hazards regression models, fitting time to first sepsis event as the outcome and lipid quartile as the exposure. We defined person-time at risk as days from first interview to first sepsis hospitalization. Participants without a sepsis event were censored at the earliest of last follow-up interview, death, or December 31, 2012. We sequentially adjusted the model for subject demographics (age, sex, race, education, income, geographic region), health behaviors (smoking or alcohol use), chronic medical conditions (cancer, chronic lung disease, CAD, diabetes, hypertension, obesity, stroke, statin use, Morisky adherence index), biomarkers (hs-CRP, cystatin C, ACR, eGFR), and functional status (weakness, exhaustion, low physical activity). We additionally examined HDL-C and LDL-C using relevant clinical cutoff values and as standardized continuous variables, both in their original forms and as a percentage of total cholesterol. We also stratified the analysis by statin use, generating new 
quartile cutoff values relative to nonusers and users. Lastly, we performed a sensitivity analysis in which several measures (body mass index, hs-CRP, cystatin C, ACR, and eGFR) were included as continuous variables.

Because of the substantial number of missing values for select variables included in our final model (HDL-C 4.8\%, LDL-C 6.1\%, cystatin C 6.8\%, hs-CRP 6.4\%, ACR $4.7 \%$, eGFR $4.3 \%$ ), we performed all modeling using multiple imputation. We implemented multiple imputation using chained equations with the Stata 'MI' suite, generating 10 imputed data sets and pooling estimates using Rubin's rules [27, 28]. Patterns of missingness were examined in order to ensure that the assumption of randomness was met and the imputation model included all variables from the final analytic model, in addition to the Nelson-Aalen cumulative hazard function (a common approach for incorporating the outcome measure) [29].We ultimately reported adjusted hazard ratios and Kaplan-Meier failure functions after pooling across imputations. All analyses were performed using Stata 13.1 (Statacorp, College Station, TX, USA).

\section{Results}

Of the 30,239 total REGARDS participants, we included 29,690 with complete follow-up information. Among this group, there were 3423 hospitalizations for serious infections, with 1845 total sepsis events among 1526 individuals. Median follow-up time was 3.6 years (IQR 1.8-5.6) for participants with first sepsis events and 6.6 years (IQR 5.2-8.1) for censored participants.

Baseline participant characteristics by lipid level quartile are presented in Table 1. Compared with the highest quartile, participants in the lowest HDL-C quartile were more likely to be male, White, have less than a high school education, reside in the stroke belt, be a current smoker, and use no alcohol. This group was also more likely to have a number of chronic medical conditions and biomarker abnormalities, including CAD, diabetes, hypertension, obesity, stroke, statin use, elevated hs-CRP/cystatinC/ACR, and reduced eGFR. Similarly, compared with the highest quartile, participants in the lowest quartile for LDL-C were more likely to be male and White; also experiencing disproportionate $\mathrm{CAD}$, diabetes, hypertension, stroke, statin use, elevated cystatin $\mathrm{C} / \mathrm{ACR}$, and reduced eGFR. In contrast to HDL-C, participants in the lowest LDL-C quartile were less likely to have elevated hs-CRP compared with the highest quartile. Significant linear trends across quartiles were observed for all biomarkers examined $(p<0.001)$.

Sepsis incidence differed substantially across HDL-C and LDL-C quartiles, with the highest incidence observed in the lowest quartile and the lowest incidence in the highest quartile for both lipid types (Table 2). The most common infection types associated with first sepsis episode were pneumonia (39.4\%), kidney and urinary tract (17.0\%), and abdominal infections (15.1\%). Pneumonia was more common for participants in the highest quartile of HDL-C compared with the lowest, while abdominal infections were more common in the lowest quartile. For LDL-C, kidney and urinary tract infections were more common in the lowest quartile compared with the highest. Compared with the higher quartiles, the lowest quartiles of HDL-C and LDL-C had higher proportions of individuals with elevated SOFA scores.

In unadjusted analyses, the lowest quartiles for both HDL-C and LDL-C were associated with increased sepsis rates (Table 3 and Fig. 1). After adjustment for demographics, health behaviors, and chronic medical conditions, HDL-C quartile remained associated with long-term risk of sepsis. However, after the addition of biomarkers to the model, HDL-C was no longer associated with rates of sepsis. Similarly, HDL-C defined using clinical cutoff values or as a continuous variable showed no associations with sepsis risk. However, LDL-C in the lowest two quartiles was associated with increased adjusted rates of sepsis. We observed a linear trend across LDL-C quartiles (Figs. 1 and 2). We obtained similar inferences when LDL-C was defined as a continuous variable or as a percentage of total cholesterol. We observed similar results when stratifying by statin use and redefining quartile cutoff values (Table 4). Lastly, we observed similar associations when adjusting for BMI, hs-CRP, Cystatin C, ACR, and eGFR as continuous variables. (Additional file 1: Table S1)

\section{Discussion}

In this large, prospective cohort study we have demonstrated that baseline cholesterol levels may be associated with long-term risk of sepsis. Specifically, we found that lower LDL-C values, though generally accepted to be favorable in terms of cardiovascular disease risk and prevention of CAD, seem to be associated with increased long-term risk of sepsis. Contrary to our original hypothesis, we found no association between low HDL-C and sepsis risk after adjusting for potential confounders.

In recent years, the bulk of sepsis research has focused on early detection and care of acute sepsis. But few efforts have conceptualized sepsis as a preventable condition. Identifying risk factors and developing a strategy for sepsis prevention could be a worthwhile endeavor to reduce the societal burden of this life-threatening and costly disease. The results of this study have important, yet perplexing implications for sepsis prevention. In the cardiovascular literature, lower LDL-C $(<130 \mathrm{mg} / \mathrm{dl})$ and higher HDL-C ( $>60 \mathrm{mg} / \mathrm{dl})$ thresholds are thought to be protective against CAD [1]. Our results, however, demonstrate that lower LDL-C levels are associated with increased long-term risk of sepsis. We have also demonstrated an 
Table 1 Baseline participant characteristics by HDL-C and LDL-C quartile

\begin{tabular}{|c|c|c|c|c|c|c|c|c|}
\hline \multirow[t]{2}{*}{ Characteristic } & \multicolumn{4}{|c|}{ HDL-C quartile } & \multicolumn{4}{|c|}{ LDL-C quartile } \\
\hline & $\begin{array}{l}\text { Q1, } \\
5-40 \\
\mathrm{mg} / \mathrm{dl}\end{array}$ & $\begin{array}{l}\text { Q2, } \\
41-49 \\
\mathrm{mg} / \mathrm{dl}\end{array}$ & $\begin{array}{l}\text { Q3, } \\
50-61 \\
\mathrm{mg} / \mathrm{dl}\end{array}$ & $\begin{array}{l}\text { Q4, } \\
62-199 \\
\mathrm{mg} / \mathrm{dl}\end{array}$ & $\begin{array}{l}\text { Q1, } \\
\text { 3-89 } \\
\mathrm{mg} / \mathrm{dl}\end{array}$ & $\begin{array}{l}\mathrm{Q} 2, \\
90-111 \\
\mathrm{mg} / \mathrm{dl}\end{array}$ & $\begin{array}{l}\text { Q3, } \\
112- \\
135 \mathrm{mg} / \mathrm{dl}\end{array}$ & $\begin{array}{l}\text { Q4, } \\
136- \\
388 \mathrm{mg} / \mathrm{dl}\end{array}$ \\
\hline Number of participants & 7305 & 7064 & 7162 & 6749 & 6984 & 7088 & 6915 & 6896 \\
\hline \multicolumn{9}{|l|}{ Demographics } \\
\hline Age (years), mean (SD) & $64.8(9.3)$ & $64.8(9.5)$ & $64.8(9.3)$ & $65.1(9.5)$ & $66.3(9.5)$ & $65.4(9.5)$ & $64.5(9.4)$ & $63.4(9.0)$ \\
\hline \multicolumn{9}{|l|}{ Age group (\%) } \\
\hline $45-49$ & 5.0 & 5.5 & 4.8 & 4.6 & 4.4 & 4.7 & 5.4 & 5.4 \\
\hline $50-59$ & 26.0 & 25.5 & 26.5 & 26.8 & 20.8 & 24.7 & 27.9 & 31.3 \\
\hline $60-69$ & 38.2 & 38.3 & 37.8 & 36.3 & 37.0 & 37.7 & 37.1 & 38.5 \\
\hline 70-79 & 24.3 & 23.4 & 24.2 & 24.6 & 28.9 & 25.1 & 23.0 & 19.8 \\
\hline$\geq 80$ & 6.6 & 7.3 & 6.6 & 7.7 & 8.8 & 7.9 & 6.7 & 5.0 \\
\hline \multicolumn{9}{|l|}{ Gender (\%) } \\
\hline Male & 71.3 & 51.6 & 35.3 & 21.7 & 51.1 & 47.1 & 43.6 & 38.9 \\
\hline Female & 28.7 & 48.4 & 64.7 & 78.3 & 48.9 & 52.9 & 56.4 & 61.1 \\
\hline \multicolumn{9}{|l|}{ Race (\%) } \\
\hline White & 67.6 & 58.8 & 55.7 & 55.5 & 62.3 & 61.3 & 58.6 & 54.6 \\
\hline Black & 32.4 & 41.3 & 44.3 & 44.5 & 37.7 & 38.7 & 41.4 & 45.5 \\
\hline \multicolumn{9}{|l|}{ Education (\%) } \\
\hline Less than high school & 13.1 & 12.8 & 12.4 & 10.9 & 13.5 & 11.8 & 11.8 & 12.1 \\
\hline High school graduate & 25.8 & 26.4 & 26.4 & 24.6 & 26.1 & 25.4 & 24.4 & 27.3 \\
\hline Some college & 27.2 & 26.6 & 26.9 & 26.5 & 26.0 & 26.7 & 27.8 & 26.7 \\
\hline College or higher & 34.0 & 34.2 & 34.3 & 38.1 & 34.5 & 36.2 & 36.1 & 33.9 \\
\hline Missing $(N)$ & 7 & 4 & 3 & 7 & 7 & 4 & 3 & 7 \\
\hline \multicolumn{9}{|l|}{ Income (\%) } \\
\hline$<\$ 20,000$ & 16.6 & 18.3 & 18.0 & 17.7 & 18.2 & 16.9 & 16.5 & 18.7 \\
\hline$\$ 20,000-34,000$ & 24.0 & 24.0 & 24.9 & 23.5 & 25.2 & 24.0 & 22.9 & 24.4 \\
\hline$\$ 35,000-74,000$ & 32.1 & 30.8 & 29.1 & 28.0 & 29.8 & 30.8 & 30.7 & 29.1 \\
\hline$\geq \$ 75,000$ & 16.6 & 15.5 & 15.5 & 16.4 & 15.0 & 16.2 & 17.3 & 15.6 \\
\hline Refused & 10.7 & 11.4 & 12.5 & 14.4 & 11.9 & 12.1 & 12.6 & 12.2 \\
\hline \multicolumn{9}{|l|}{ Geographic region (\%) } \\
\hline Stroke buckle & 20.7 & 20.6 & 21.7 & 20.9 & 22.4 & 20.7 & 20.4 & 20.2 \\
\hline Stroke belt & 37.0 & 35.7 & 33.7 & 32.6 & 35.1 & 35.1 & 33.7 & 34.9 \\
\hline Nonbelt/buckle & 42.3 & 43.7 & 44.7 & 46.5 & 42.5 & 44.2 & 46.0 & 44.9 \\
\hline \multicolumn{9}{|l|}{ Health behaviors } \\
\hline \multicolumn{9}{|l|}{ Smoking status (\%) } \\
\hline Current & 17.1 & 15.1 & 13.2 & 12.1 & 14.1 & 13.1 & 14.2 & 15.8 \\
\hline Past & 44.9 & 41.1 & 38.3 & 37.1 & 44.4 & 40.9 & 39.1 & 36.9 \\
\hline Never & 38.1 & 43.9 & 48.5 & 50.9 & 41.5 & 46.0 & 46.7 & 47.2 \\
\hline Missing $(N)$ & 17 & 29 & 31 & 28 & 23 & 24 & 31 & 25 \\
\hline \multicolumn{9}{|l|}{ Alcohol use (\%) } \\
\hline Heavy & 1.8 & 3.1 & 4.0 & 7.5 & 4.0 & 4.4 & 3.9 & 3.7 \\
\hline Moderate & 31.8 & 32.5 & 33.5 & 36.7 & 32.3 & 35.0 & 34.8 & 32.0 \\
\hline None & 66.4 & 64.4 & 62.5 & 55.9 & 63.7 & 60.6 & 61.3 & 64.3 \\
\hline Missing (N) & 155 & 153 & 106 & 134 & 145 & 140 & 122 & 130 \\
\hline
\end{tabular}


Table 1 Baseline participant characteristics by HDL-C and LDL-C quartile (Continued)

\begin{tabular}{|c|c|c|c|c|c|c|c|c|}
\hline \multicolumn{9}{|l|}{ Chronic medical conditions } \\
\hline Cancer (\%) & 10.3 & 8.5 & 8.0 & 8.0 & 10.2 & 9.3 & 8.0 & 7.2 \\
\hline Chronic lung disease (\%) & 8.3 & 9.2 & 9.2 & 10.0 & 10.7 & 9.7 & 8.8 & 7.4 \\
\hline Coronary artery disease (\%) & 25.3 & 19.2 & 15.1 & 11.5 & 28.7 & 18.7 & 12.9 & 10.7 \\
\hline Diabetes (\%) & 30.3 & 25.4 & 19.0 & 14.2 & 33.5 & 22.9 & 17.0 & 14.8 \\
\hline Hypertension (\%) & 63.5 & 60.8 & 58.0 & 53.7 & 67.0 & 60.2 & 55.9 & 52.3 \\
\hline Obesity (\%) & 61.7 & 57.2 & 53.6 & 40.0 & 54.5 & 52.0 & 52.3 & 54.0 \\
\hline Stroke (\%) & 7.6 & 7.0 & 5.7 & 4.4 & 8.5 & 6.3 & 5.0 & 4.9 \\
\hline Statin use (\%) & 35.3 & 35.2 & 31.6 & 23.6 & 56.6 & 47.0 & 36.8 & 37.0 \\
\hline \multicolumn{9}{|l|}{ Biomarkers } \\
\hline $\mathrm{hs}-\mathrm{CRP}>3.0 \mathrm{mg} / \mathrm{dl}$ & 44.6 & 41.8 & 40.3 & 35.5 & 38.3 & 39.6 & 40.8 & 43.8 \\
\hline Missing $(N)$ & 148 & 165 & 167 & 179 & 150 & 177 & 159 & 160 \\
\hline Cystatin C> $1.12 \mathrm{mg} / \mathrm{dl}$ & 34.1 & 26.7 & 21.6 & 17.3 & 32.1 & 25.7 & 22.2 & 19.7 \\
\hline Missing $(N)$ & 183 & 187 & 208 & 212 & 189 & 199 & 196 & 191 \\
\hline ACR > $30 \mu \mathrm{g} / \mathrm{mg}(\%)$ & 18.8 & 15.7 & 13.0 & 12.5 & 17.5 & 15.0 & 13.7 & 13.3 \\
\hline Missing $(N)$ & 267 & 308 & 249 & 254 & 295 & 287 & 227 & 250 \\
\hline $\mathrm{eGFR}<60 \mathrm{ml} / \mathrm{min} / 1.73 \mathrm{~m}^{2}$ & 14.7 & 11.8 & 10.3 & 8.7 & 15.2 & 11.9 & 9.7 & 8.8 \\
\hline Missing $(N)$ & 1 & 0 & 1 & 0 & 0 & 0 & 1 & 1 \\
\hline \multicolumn{9}{|l|}{ Functional status } \\
\hline Weakness (SF-12 PCS < 75) (\%) & 31.0 & 29.9 & 28.8 & 26.7 & 33.8 & 28.9 & 26.5 & 26.8 \\
\hline Reported exhaustion (\%) & 15.7 & 13.9 & 13.4 & 11.7 & 14.8 & 14.1 & 12.4 & 13.0 \\
\hline Low physical activity (\%) & 34.7 & 33.5 & 33.6 & 32.6 & 35.4 & 33.7 & 32.6 & 32.6 \\
\hline
\end{tabular}

Total of 29,690 participants. LDL-C measurement missing for 1807 participants. HDL-C measurement missing for 1410 participants All $p<0.05$ based on Pearson chi-square tests of association

$L D L-C$ low-density lipoprotein, $H D L-C$ high-density lipoprotein, $S D$ standard deviation, $h s-C R P$ high-sensitivity $C$-reactive protein, $A C R$ albumin-creatinine ratio, eGFR estimated glomerular filtration rate, SF-12 12-item short form survey, PCS physical composite score

association between low LDL-C and HDL-C and elevated SOFA scores.

Previous studies have demonstrated that low cholesterol levels are a risk factor for sepsis [16, 30, 31] and have also shown that lipid levels are rapidly changing during early sepsis and may predict outcomes [12-14, 30, 31]. In the setting of infection, cholesterol levels may drop dramatically because of decreases in LDL-C and HDL-C. The exact mechanism for this is unknown; however, bacterial endotoxin, tumor necrosis factor, interleukin-2, and interferon beta, all potentially present during systemic infection, reduce serum cholesterol levels [32-37]. Additionally, both LDL-C and HDL-C play a proven role in clearance of bacterial toxins, lipopolysaccharide (LPS) from Gram-negative bacteria, and lipoteichoic acid from Gram-positive bacteria. In studies where LPS was added to human whole blood in vitro, LPS was bound to HDL-C (60\%), LDL-C (25\%), and very low density lipoprotein (VLDL)-cholesterol (12\%) [38]. Therefore, one potential explanation for the increased long-term sepsis risk with low LDL-C is the inability to clear bacterial toxins from the bloodstream.
Interestingly, our study did not demonstrate an association between low HDL-C and long-term sepsis risk. At a glance, these results appear contrary to findings by Chien et al. that low HDL-C levels in acute sepsis are predictive of increased 30-day mortality, prolonged ICU stay ( $>7$ days), and increased hospital-acquired infections. However, there are two reasons why these results should not be entirely surprising. First, recent evidence has demonstrated that HDL-C function may be more important than quantity. Examples include recent clinical trials that failed to show benefit to medically elevating HDL-C to prevent myocardial infarction [3]. Another recent Mendelian randomization study failed to find a reduced risk of myocardial infarction in a group of patients with a specific single nucleotide polymorphism predisposing to elevated HDL-C [39]. These studies are timely, given a burgeoning area of research demonstrating that HDL-C can become dysfunctional and proinflammatory under certain chronic (metabolic syndrome, diabetes mellitus, rheumatoid arthritis, CAD) and acute (sepsis, myocardial infarction, stroke) inflammatory conditions [40-44]. HDL-C function may then 
Table 2 Sepsis incidence rates and hospitalization characteristics by HDL-C and LDL-C quartile

\begin{tabular}{|c|c|c|c|c|c|c|c|c|c|c|}
\hline \multirow[t]{2}{*}{ Variable } & \multicolumn{4}{|l|}{$\mathrm{HDL}-\mathrm{C}$ quartile } & \multirow[t]{2}{*}{$p$} & \multicolumn{4}{|l|}{ LDL-C quartile } & \multirow[t]{2}{*}{$p$} \\
\hline & $\begin{array}{l}\text { Q1, } \\
5-40 \\
\mathrm{mg} / \mathrm{dl}\end{array}$ & $\begin{array}{l}\mathrm{Q} 2, \\
41-49 \\
\mathrm{mg} / \mathrm{dl}\end{array}$ & $\begin{array}{l}\text { Q3, } \\
50-61 \\
\mathrm{mg} / \mathrm{dl}\end{array}$ & $\begin{array}{l}\mathrm{Q} 4, \\
62-199 \\
\mathrm{mg} / \mathrm{dl}\end{array}$ & & $\begin{array}{l}\text { Q1, } \\
3-89 \\
\mathrm{mg} / \mathrm{dl}\end{array}$ & $\begin{array}{l}\text { Q2, } \\
90-111 \\
\mathrm{mg} / \mathrm{dl}\end{array}$ & $\begin{array}{l}\mathrm{Q} 3 \\
112-135 \\
\mathrm{mg} / \mathrm{dl}\end{array}$ & $\begin{array}{l}\text { Q4, } \\
136-388 \\
\mathrm{mg} / \mathrm{dl}\end{array}$ & \\
\hline $\begin{array}{l}\text { Number of } \\
\text { participants }\end{array}$ & 7305 & 7064 & 7162 & 6749 & & 6984 & 7088 & 6915 & 6896 & \\
\hline Sepsis events, $N(\%)$ & $485(6.6)$ & $372(5.3)$ & $329(4.6)$ & $264(3.9)$ & & $451(6.5)$ & $399(5.6)$ & $304(4.4)$ & $261(3.8)$ & \\
\hline $\begin{array}{l}\text { IR (per } 1000 \\
\text { person-years), 95\% Cl }\end{array}$ & $10.6(9.7-11.6)$ & $8.5(7.7-9.4)$ & $7.3(6.6-8.1)$ & $6.2(5.5-7.0)$ & & $10.6(9.6-11.6)$ & $8.9(8.1-9.9)$ & $6.9(6.2-7.7)$ & $6.0(5.3-6.8)$ & \\
\hline \multicolumn{11}{|c|}{ Sepsis event hospitalization characteristics } \\
\hline \multicolumn{11}{|l|}{ Infection type, N (\%) } \\
\hline Pneumonia & $179(36.9)$ & $128(34.4)$ & $138(42.0)$ & $120(45.5)$ & 0.018 & $181(40.1)$ & $151(37.8)$ & $113(37.2)$ & $105(40.2)$ & 0.79 \\
\hline $\begin{array}{l}\text { Kidney and } \\
\text { urinary tract } \\
\text { infections }\end{array}$ & $86(17.7)$ & $71(19.1)$ & $58(17.6)$ & $22(12.5)$ & 0.16 & $80(17.7)$ & 79 (19.8) & $61(20.1)$ & $22(8.4)$ & $<0.001$ \\
\hline Abdominal & $78(16.1)$ & $71(19.1)$ & 40 (12.2) & $22(12.5)$ & 0.038 & $62(13.8)$ & 64 (16.0) & $44(14.5)$ & 47 (18.0) & 0.45 \\
\hline $\begin{array}{l}\text { Skin and soft } \\
\text { tissue }\end{array}$ & $50(10.3)$ & $29(7.8)$ & $17(5.2)$ & $20(7.6)$ & 0.066 & $43(9.5)$ & $26(6.5)$ & $26(8.6)$ & $19(7.3)$ & 0.40 \\
\hline $\begin{array}{l}\text { Bronchitis, } \\
\text { influenza and } \\
\text { other lung } \\
\text { infections }\end{array}$ & $34(7.0)$ & $34(9.1)$ & 35 (10.6) & $31(11.7)$ & 0.13 & $34(7.5)$ & $38(9.5)$ & $34(11.2)$ & $27(10.3)$ & 0.35 \\
\hline Sepsis & $42(8.7)$ & $21(5.7)$ & $18(5.5)$ & $16(6.1)$ & 0.20 & $30(6.7)$ & $23(5.8)$ & $16(5.3)$ & $24(9.2)$ & 0.24 \\
\hline $\begin{array}{l}\text { Fever of } \\
\text { unknown origin }\end{array}$ & $4(0.8)$ & $7(1.9)$ & $11(3.3)$ & $4(1.5)$ & 0.072 & $6(1.3)$ & $9(2.3)$ & $3(1.0)$ & $7(2.7)$ & 0.34 \\
\hline Surgical wound & $1(0.2)$ & $3(0.8)$ & $5(1.5)$ & $1(0.4)$ & 0.14 & $2(0.4)$ & $2(0.5)$ & $2(0.7)$ & $4(1.5)$ & 0.41 \\
\hline $\begin{array}{l}\text { Catheter (IV/ } \\
\text { central/dialysis) }\end{array}$ & $2(0.4)$ & $0(0)$ & $0(0)$ & $3(1.1)$ & - & $2(0.4)$ & $1(0.3)$ & $1(0.3)$ & $0(0)$ & 0.91 \\
\hline Meningitis & $0(0)$ & $2(0.5)$ & $2(0.6)$ & $1(0.4)$ & 0.27 & $2(0.4)$ & $1(0.3)$ & $0(0)$ & $2(0.8)$ & 0.51 \\
\hline Unknown/other & $9(1.9)$ & $6(1.6)$ & $5(1.5)$ & $2(0.8)$ & 0.70 & $9(2.0)$ & $5(1.3)$ & $4(1.3)$ & $4(1.5)$ & 0.85 \\
\hline $\begin{array}{l}\text { Elevated SOFA score } \\
(\text { SOFA } \geq 2), N(\%)\end{array}$ & $266(54.9)$ & $175(47.0)$ & $147(44.7)$ & $119(45.1)$ & 0.010 & $251(55.7)$ & $201(50.4)$ & $127(41.8)$ & $107(41.0)$ & $<0.001$ \\
\hline $\begin{array}{l}\text { Admitted to ICU vs } \\
\text { floor, } N(\%)^{\mathrm{a}}\end{array}$ & $69(16.0)$ & $42(12.8)$ & $41(14.4)$ & $24(9.9)$ & 0.16 & $70(17.0)$ & 41 (11.6) & $26(10.2)$ & $32(13.9)$ & 0.051 \\
\hline $\begin{array}{l}\text { In-hospital death, } \\
N(\%)\end{array}$ & $43(8.9)$ & $30(8.1)$ & $30(9.1)$ & $25(9.5)$ & 0.93 & $43(9.5)$ & $35(8.8)$ & $19(6.3)$ & $29(11.1)$ & 0.22 \\
\hline
\end{tabular}

Total of 29,690 participants. LDL-C measurement missing for 1807 participants. HDL-C measurement missing for 1410 participants

$p$ values from Pearson chi-square tests of association for variables with at least five observations in $75 \%$ of table cells, and Fisher exact tests for all others ancludes only events resulting in an inpatient admission and those with inpatient records available

$L D L-C$ low-density lipoprotein, HDL-C high-density lipoprotein, IR incidence rate, $C l$ confidence interval, SOFA Sepsis-related Organ Failure Assessment,

ICU intensive care unit

explain the lack of a protective effect in this study, given the prevalence of chronic inflammatory conditions within the REGARDS cohort. Another explanation may be the difference between rapid lowering of lipid levels during acute inflammatory states which relate to disease severity, rather than chronically low levels of HDL-C which, if dysfunctional, may not protect as well against sepsis. In order to address this question, future studies aimed at prospectively measuring dysfunctional HDL and monitoring for the development of sepsis are needed.
Potential clinical applications for the results of this study may include modulation of LDL clearance. Lipid-based pathogens from bacteria are first bound to binding proteins which are then bound by HDL and transferred to LDL or VLDL molecules. LDL and VLDL are then cleared from the bloodstream by hepatocytes via the LDL receptor. The proprotein convertase subtilisin/kexin type 9 (PCSK9) molecule binds the LDL receptor on hepatocytes, and promotes internalization and lysosomal degradation of the receptor, thus preventing clearance of LDL and VLDL 


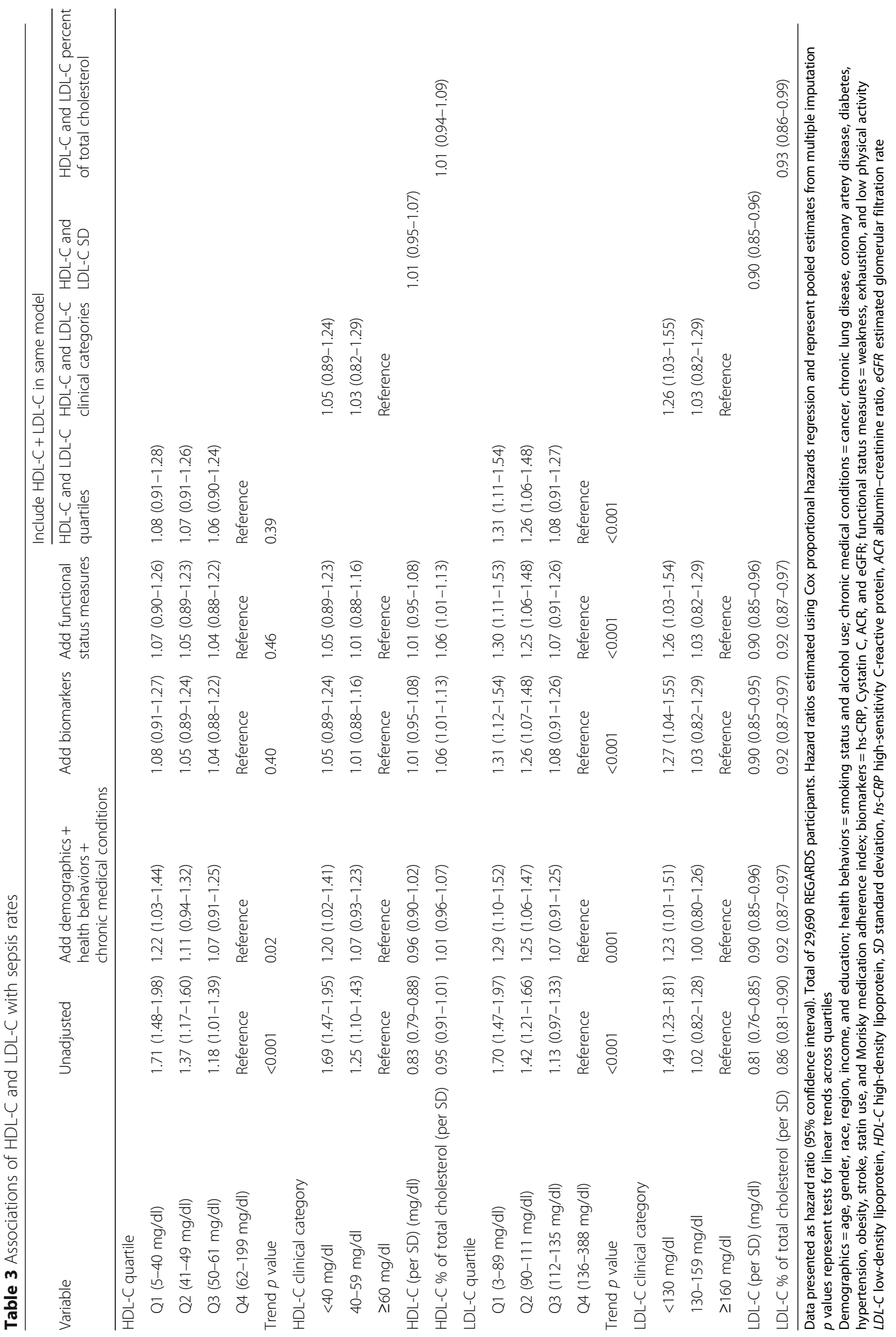



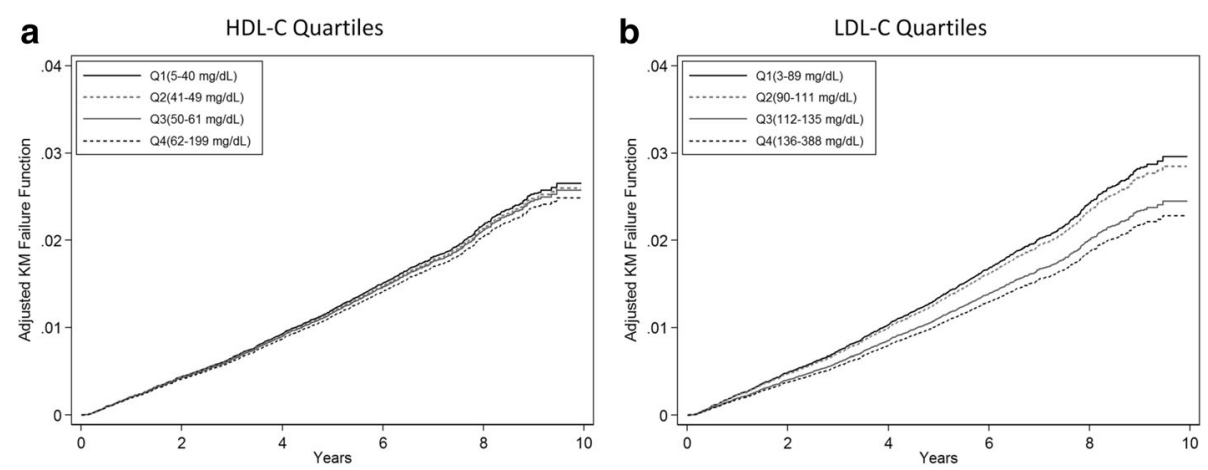

Fig. 1 Adjusted Kaplan-Meier (KM) failure curves for time to sepsis by HDL-C and LDL-C quartiles (Q1 = lowest quartile, Q4 = highest quartile). Total of 29,690 participants. (a) High-density lipoprotein cholesterol (HDL-C) and (b) low-density lipoprotein cholesterol (LDL-C). All failure functions estimated among White, female, nonsmoking and non-alcohol-consuming participants residing in the nonbelt region, with no history of comorbidities, normal biomarker levels, and no functional status impairments (all binary variables set to zero and all categorical variables set to the reference groups)

from the blood [45]. PCSK9 inhibitors are currently under investigation as lipid-lowering agents because they promote LDL clearance and thus reduce LDL levels, but they may also have an application in clearance of lipid-based pathogens in sepsis. PCSK9 inhibition results in decreased inflammatory cytokine production and physiological responses to endotoxin in septic mice and increased PCSK9 levels are associated with reduced endotoxin clearance and organ failure in sepsis $[45,46]$. Although this is a new area of research, it demonstrates that modulation of cholesterol metabolism may have potential as a future application of these findings. In addition, because HDL plays a critical intermediary step in lipid-based pathogen clearance, investigating HDL function in sepsis is of increased importance. If the association between HDL-C and sepsis-associated organ dysfunction is valid, studies of HDL-based therapies including apoliprotein mimetic peptides and synthetic HDL may hold potential $[47,48]$.

In our analysis, we chose to stratify by baseline statin use, because statins have had significant attention in the literature with regards to protection against sepsis. The mechanisms by which statins may protect against sepsis are unknown, though a new class of lipid mediators called resolvins may play a role [49]. Also, varying protection against sepsis has been found between different statins, with atorvastatin being the most promising [50]. Our group completed a recent study which did not find baseline statin use to be protective against long-term sepsis in the REGARDS cohort [51]. In addition, statin use did not significantly influence our results with regards to baseline lipid levels. Two recent meta-analyses of only randomized, controlled trials also found no benefit to statin use in sepsis $[52,53]$.
This study had several limitations. Only baseline lipid levels were available for analysis, there were no repeat levels or levels prior to the sepsis event. Therefore, lipid levels may have changed over time prior to the sepsis event, though we feel that the magnitude of change would have been unlikely to change our results. REGARDS was not designed to study sepsis, and therefore some sepsis events could have been missed. However, given the systematic hospital record review, we believe we have minimized the potential for missed or wrongly identified cases of sepsis. The REGARDS cohort only includes Black and White participants at least 45 years of age, and therefore results may not be as generalizable to other ethnic groups or a younger population. Sepsis events were limited to community-acquired sepsis and did not include hospital-acquired sepsis events, because hospital sepsis is more likely to be influenced by multiple factors related to the hospitalization. Also, while we made every effort to adjust for potential confounders, other variables such as vaccination or access to healthcare which were not accounted for may have influenced the results. Finally, residual confounding is a potential concern, as with any observational study. We accounted for a comprehensive range of variables through our risk adjustment strategy for each participant at the beginning of the REGARDS study, but we could not account for changes in these patterns over time.

\section{Conclusions}

In this large, prospective cohort study, low LDL-C was associated with increased long-term rates of community-acquired sepsis. HDL-C was not associated with long-term sepsis. Future studies should attempt to characterize the pathophysiologic or mechanistic basis for these associations. 
Table 4 Associations of HDL-C and LDL-C with sepsis rates stratified by statin use

\begin{tabular}{|c|c|c|c|c|c|}
\hline \multirow[t]{3}{*}{ Variable } & \multirow[t]{3}{*}{ Total N } & \multirow{3}{*}{$\begin{array}{l}\text { Sepsis events, } \\
N(\%)\end{array}$} & \multirow{3}{*}{$\begin{array}{l}\text { Sepsis incidence } \\
\text { (per } 1000 \text { person-years), } \\
\text { IR }(95 \% \mathrm{Cl})\end{array}$} & \multicolumn{2}{|c|}{ Models for sepsis hazard } \\
\hline & & & & \multirow{2}{*}{$\begin{array}{l}\text { Unadjusted, } \\
\mathrm{HR}(95 \% \mathrm{Cl})\end{array}$} & \multirow{2}{*}{$\begin{array}{l}\text { Adjusted }^{\mathrm{a}} \text {, } \\
\text { HR (95\% Cl) }\end{array}$} \\
\hline & & & & & \\
\hline \multicolumn{6}{|l|}{ Statin non-users } \\
\hline \multicolumn{6}{|l|}{ HDL-C quartile } \\
\hline Q1 (5-41 mg/dl) & 5233 & $315(6.0)$ & $9.6(8.6-10.7)$ & $1.73(1.43-2.09)$ & $1.09(0.88-1.36)$ \\
\hline Q2 (42-50 mg/dl) & 4540 & $223(4.9)$ & $7.9(6.9-9.0)$ & $1.43(1.16-1.76)$ & $1.11(0.89-1.37)$ \\
\hline Q3 (51-62 mg/dl) & 4751 & $201(4.2)$ & $6.8(5.9-7.8)$ & $1.21(0.98-1.49)$ & $1.06(0.86-1.31)$ \\
\hline \multirow[t]{2}{*}{ Q4 (63-199 mg/dl) } & 4837 & $169(3.5)$ & $5.5(4.8-6.4)$ & Reference & Reference \\
\hline & & & Trend $p$ value & $<0.001$ & 0.44 \\
\hline \multicolumn{6}{|l|}{ LDL-C quartile } \\
\hline Q1 (3-99 mg/dl) & 4816 & $278(5.8)$ & $9.4(8.3-10.5)$ & $1.54(1.28-1.86)$ & $1.32(1.10-1.60)$ \\
\hline Q2 (100-120 mg/dl) & 4810 & $231(4.8)$ & $7.6(6.7-8.7)$ & $1.29(1.06-1.57)$ & $1.19(0.98-1.46)$ \\
\hline Q3 (121-143 mg/dl) & 4842 & $212(4.4)$ & $6.9(6.0-7.9)$ & $1.13(0.93-1.37)$ & $1.11(0.91-1.36)$ \\
\hline \multirow[t]{2}{*}{ Q4 (144-388 mg/dl) } & 4619 & $169(3.7)$ & $5.8(5.0-6.8)$ & Reference & Reference \\
\hline & & & Trend $p$ value & $<0.001$ & 0.003 \\
\hline \multicolumn{6}{|l|}{ Statin users } \\
\hline \multicolumn{6}{|l|}{ HDL-C quartile } \\
\hline Q1 (11-39 mg/dl) & 2304 & $170(7.4)$ & $11.8(10.2-13.7)$ & $1.37(1.08-1.73)$ & $0.89(0.69-1.17)$ \\
\hline Q2 (40-47 mg/dl) & 2274 & $134(5.9)$ & $9.6(8.1-11.4)$ & $1.11(0.87-1.43)$ & $0.87(0.67-1.13)$ \\
\hline Q3 (48-57 mg/dl) & 2163 & $119(5.5)$ & $8.8(7.3-10.5)$ & $1.03(0.80-1.33)$ & $0.93(0.72-1.20)$ \\
\hline \multirow[t]{2}{*}{ Q4 (58-156 mg/dl) } & 2178 & $119(5.5)$ & $8.7(7.3-10.4)$ & Reference & Reference \\
\hline & & & Trend $p$ value & 0.006 & 0.39 \\
\hline \multicolumn{6}{|l|}{ LDL-C quartile } \\
\hline Q1 (11-76 mg/dl) & 2210 & $175(7.9)$ & $13.1(11.3-15.1)$ & $2.00(1.55-2.59)$ & $1.50(1.14-1.96)$ \\
\hline Q2 (77-93 mg/dl) & 2304 & $142(6.2)$ & $9.9(8.4-11.7)$ & $1.55(1.19-2.03)$ & $1.36(1.03-1.78)$ \\
\hline Q3 (94-111 mg/dl) & 2091 & $127(6.1)$ & $9.6(8.1-11.4)$ & $1.49(1.13-1.96)$ & $1.38(1.04-1.81)$ \\
\hline \multirow[t]{2}{*}{ Q4 (112-315 mg/dl) } & 2191 & $81(3.7)$ & $5.9(4.7-7.3)$ & Reference & Reference \\
\hline & & & Trend $p$ value & $<0.001$ & 0.008 \\
\hline
\end{tabular}

Total of 29,690 REGARDS participants. HRs estimated using Cox proportional hazards regression and represent pooled estimates from multiple imputation $p$ values represent tests for linear trends across quartiles

${ }^{a}$ Adjusted for demographics, health behaviors, chronic medical conditions, biomarkers, and functional status measures. Demographics = age, gender, race, region, income, and education; health behaviors = smoking status and alcohol use; chronic medical conditions = cancer, chronic lung disease, coronary artery disease, diabetes, hypertension, obesity, stroke, and Morisky medication adherence index; biomarkers = hs-CRP, Cystatin C, ACR, and eGFR; functional status measures = weakness, exhaustion, and low physical activity

$H R$ hazard ratio, $I R$ incidence rate, $C l$ confidence interval, $L D L-C$ low-density lipoprotein, $H D L-C$ high-density lipoprotein, $h s-C R P$ high-sensitivity $C$-reactive protein, $A C R$ albumin-creatinine ratio, eGFR estimated glomerular filtration rate

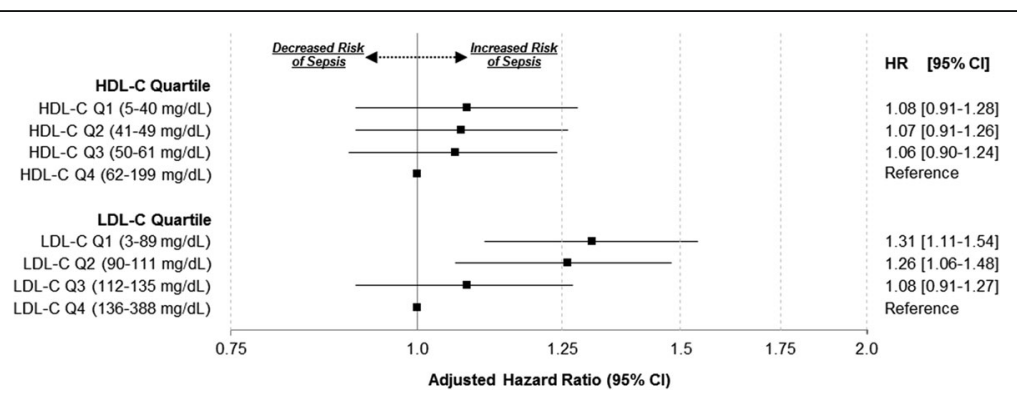

Fig. 2 Risk of sepsis by HDL-C or LDL-C quartile (Q1 = lowest quartile, Q4 = highest quartile). LDL-C low-density lipoprotein, HDL-C high-density lipoprotein, HR hazard ratio, Cl confidence interval 


\section{Additional file}

Additional file 1: Table S1. Is presenting results analyzed using a nonimputed data set. *Adjusted for demographics, health behaviors, chronic medical conditions, biomarker values, and functional status measures. Demographics = age, gender, race, region, income, and education; health behaviors = smoking status and alcohol use; chronic medical conditions = cancer, chronic lung disease, coronary artery disease, diabetes, hypertension, obesity, stroke, and Morisky medication adherence index; biomarkers = hs-CRP, Cystatin C, ACR, and eGFR; functional status measures = weakness, exhaustion, and low physical activity. HR hazard ratio, Cl confidence interval, LDL-C low-density lipoprotein cholesterol, HDL-C high-density lipoprotein cholesterol, hs-CRP high-sensitivity C-reactive protein, $A C R$ albumin-creatinine ratio, eGFR estimated glomerular filtration rate. (DOCX $12 \mathrm{~kb}$ )

\section{Abbreviations}

ACR: Urinary albumin-to-creatinine ratio; CAD: Coronary artery disease; ED: Emergency department; eGFR: Estimated glomerular filtration rate; HDLC: High-density lipoprotein cholesterol; hs-CRP: High-sensitivity C-reactive protein; IQR: Interquartile range; LDL-C: Low-density lipoprotein cholesterol; PCS: Physical composite score; PCSK9: Proprotein convertase subtilisin/kexin type 9 molecule; REGARDS: Reasons for Geographic and Racial Disparities in Stroke; SF-12: 12-Item Short Form Health Survey; SIRS: Systemic inflammatory response syndrome criteria; SOFA: Sequential (sepsis-related) Organ Failure Assessment; VLDL: very low density lipoprotein

\section{Acknowledgements}

The authors thank the other investigators, the staff, and the participants of the REGARDS study for their valuable contributions. A full list of participating REGARDS investigators and institutions can be found online: http:// www.regardsstudy.org and http://www.regardssepsis.org.

\section{Funding}

This study was supported by award R01-NR012726 from the National Institute for Nursing Research, UL1-RR025777 from the National Center for Research Resources, as well as by grants from the Center for Clinical and Translational Science and the Lister Hill Center for Health Policy of the University of Alabama at Birmingham. The parent REGARDS study was supported by cooperative agreement U01-NS041588 from the National Institute of Neurological Disorders and Stroke, National Institutes of Health, Department of Health and Human Service. The content is solely the responsibility of the authors and does not necessarily represent the official views of the funding agencies. Representatives of the funding agencies have been involved in the review of the manuscript but not directly involved in the collection, management, analysis, or interpretation of the data.

JPD is currently supported by grant T32-HS013852 from the Agency for Healthcare Research and Quality, Rockville, MD, USA.

\section{Availability of data and materials}

Supporting data from the REGARDS Sepsis study can be obtained by emailing the corresponding author (HEW).

\section{Authors' contributions}

FWG, HEW, and SD devised the study. HEW, GH, and MMS supervised the data collection and chart reviews. HEW, JPD, SD, GH, MMS, and EBL provided methodological and statistical advice on study design and data analysis. $\mathrm{SD}, \mathrm{MMS}$, and $\mathrm{EBL}$ provided expertise in cardiovascular disease and epidemiology. FWG, JPD, and HEW drafted the manuscript and all authors contributed substantially to its revision. All authors read and approved the manuscript.

\section{Authors' information}

Not applicable.

\section{Competing interests}

MMS reports the following potential conflicts of interest: Amgen, salary support to study patterns of statin use in Medicare and other large databases; diaDexus, salary support for a research grant on lipids and CAD outcomes; diaDexus, consulting to help with FDA application. EBL reports salary support and consulting income from Amgen and consulting income from Robinson Calcagnie Robinson Shapiro Davis. The remaining authors do not report any related conflicts of interest. All authors have read Biomed Central's section on competing interests and have no competing interests to disclose.

\section{Consent for publication}

All authors have provided consent for publication of the manuscript.

\section{Ethical approval and consent to participate}

The REGARDS study was approved by the institutional review boards (University of Alabama at Birmingham) of participating institutions, and all participants provided verbal consent before the telephone interview and written informed consent before the in-home study visit.

\section{Author details}

${ }^{1}$ Department of Emergency Medicine, University of Florida College of Medicine, Jacksonville, FL, USA. ²Department of Emergency Medicine, University of Alabama School of Medicine, Birmingham, AL, USA. ${ }^{3}$ Department of Epidemiology, University of Alabama at Birmingham, Birmingham, AL, USA. ${ }^{4}$ Department of Medicine, University of Alabama School of Medicine, Birmingham, AL, USA. ${ }^{5}$ Department of Medicine, Weill Cornell Medical College, New York, NY, USA. ${ }^{6}$ Department of Epidemiology, University of Florida, Gainesville, FL, USA. ${ }^{7}$ Department of Family Medicine, Mayo Clinic, Jacksonville, FL, USA. ${ }^{8}$ Department of Biostatistics, University of Alabama at Birmingham, Birmingham, AL, USA. ${ }^{9}$ Department of Emergency Medicine, University of Alabama at Birmingham, 619 19th Street South, OHB 251, Birmingham, AL 35249, USA.

Received: 22 July 2016 Accepted: 25 November 2016

\section{Published online: 23 December 2016}

\section{References}

1. Stone NJ, et al. 2013 ACC/AHA Guideline on the Treatment of Blood Cholesterol to Reduce Atherosclerotic Cardiovascular Risk in Adults: a report of the American College of Cardiology/American Heart Association Task Force on Practice Guidelines. J Am Coll Cardiol. 2014;63:2889-934.

2. Wilson PW, Abbott RD, Castelli WP. High density lipoprotein cholesterol and mortality, The Framingham Heart Study. Arteriosclerosis. 1988;8:737-41.

3. Boden WE, et al. Niacin in patients with low HDL cholesterol levels receiving intensive statin therapy. N Engl J Med. 2011;365:2255-67.

4. Wang HE, Shapiro NI, Angus DC, Yealy DM. National estimates of severe sepsis in United States emergency departments. Crit Care Med. 2007:35:1928-36.

5. Angus DC, Wax RS. Epidemiology of sepsis: an update. Crit Care Med. 2001:29:S109-16.

6. Angus DC, et al. Epidemiology of severe sepsis in the United States: analysis of incidence, outcome, and associated costs of care. Crit Care Med. 2001;29:1303-10.

7. Catapano AL, Pirillo A, Bonacina F, Norata GD. HDL in innate and adaptive immunity. Cardiovasc Res. 2014;103:372-83.

8. Khovidhunkit W, et al. Effects of infection and inflammation on lipid and lipoprotein metabolism: mechanisms and consequences to the host. J Lipid Res. 2004;45:1169-96.

9. Murphy AJ, et al. High-density lipoprotein reduces the human monocyte inflammatory response. Arterioscler Thromb Vasc Biol. 2008;28:2071-7.

10. Murphy AJ, et al. Neutrophil activation is attenuated by high-density lipoprotein and apolipoprotein A-I in in vitro and in vivo models of inflammation. Arterioscler Thromb Vasc Biol. 2011;31:1333-41.

11. Heitzer T, Schlinzig T, Krohn K, Meinertz T, Münzel T. Endothelial dysfunction, oxidative stress, and risk of cardiovascular events in patients with coronary artery disease. Circulation. 2001;104:2673-8.

12. Chien J-Y, Jerng J-S, Yu C-J, Yang P-C. Low serum level of high-density lipoprotein cholesterol is a poor prognostic factor for severe sepsis. Crit Care Med. 2005;33:1688-93. 
13. Chien Y-F, Chen C-Y, Hsu C-L, Chen K-Y, Yu C-J. Decreased serum level of lipoprotein cholesterol is a poor prognostic factor for patients with severe community-acquired pneumonia that required intensive care unit admission. J Crit Care. 2015;30:506-10.

14. van Leeuwen $\mathrm{HJ}$, et al. Lipoprotein metabolism in patients with severe sepsis. Crit Care Med. 2003;31:1359-66.

15. Feingold KR, Grunfeld C. Lipoproteins: are they important components of host defense? Hepatology. 1997;26:1685-6.

16. Lagrost $\mathrm{L}$, et al. Low preoperative cholesterol level is a risk factor of sepsis and poor clinical outcome in patients undergoing cardiac surgery with cardiopulmonary bypass. Crit Care Med. 2014;42:1065-73.

17. Wang $\mathrm{HE}$, et al. Chronic medical conditions and risk of sepsis. PLoS One. 2012;7:e48307.

18. Howard VJ, et al. The reasons for geographic and racial differences in stroke study: objectives and design. Neuroepidemiology. 2005;25:135-43.

19. Patel MR, et al. ACCF/SCAI/STS/AATS/AHA/ASNC/HFSA/SCCT 2012 appropriate use criteria for coronary revascularization focused update: a report of the American College of Cardiology Foundation Appropriate Use Criteria Task Force, Society for Cardiovascular Angiography and In. J Thorac Cardiovasc Surg. 2012;143:780-803.

20. Morisky DE, Green LW, Levine DM. Concurrent and predictive validity of a self-reported measure of medication adherence. Med Care. 1986;24:67-74.

21. Johansen KL, Chertow GM, Jin C, Kutner NG. Significance of frailty among dialysis patients. J Am Soc Nephrol. 2007;18:2960-7.

22. Johansen $\mathrm{KL}$, et al. Comparison of self-report-based and physical performance-based frailty definitions among patients receiving maintenance hemodialysis. Am J Kidney Dis. 2014;64:600-7.

23. Woods NF, et al. Frailty: emergence and consequences in women aged 65 and older in the Women's Health Initiative observational study. J Am Geriatr Soc. 2005;53:1321-30.

24. Vincent $J \mathrm{~L}$, et al. The SOFA (Sepsis-related Organ Failure Assessment) score to describe organ dysfunction/failure. On behalf of the Working Group on Sepsis-Related Problems of the European Society of Intensive Care Medicine. Intensive Care Med. 1996;22:707-10.

25. Singer $M$, et al. The Third International Consensus Definitions for Sepsis and Septic Shock (Sepsis-3). JAMA. 2016;315:801.

26. Friedewald WT, Levy RI, Fredrickson DS. Estimation of the concentration of low-density lipoprotein cholesterol in plasma, without use of the preparative ultracentrifuge. Clin Chem. 1972;18:499-502.

27. Rubin DB, Schenker N. Multiple imputation in health-care databases: an overview and some applications. Stat Med. 1991;10:585-98.

28. Rubin DB. Multiple imputation after 18+ years. J Am Stat Assoc. 1996;91:473-89.

29. White IR, Royston P. Imputing missing covariate values for the Cox model. Stat Med. 2009;28:1982-98.

30. Grion CM, et al. Lipoproteins and CETP levels as risk factors for severe sepsis in hospitalized patients. Eur J Clin Invest. 2010;40:330-8.

31. Lee $\mathrm{SH}$, et al. Prognostic implications of serum lipid metabolism over time during sepsis. Biomed Res Int. 2015;2015:789298.

32. Auerbach BJ, Parks JS. Lipoprotein abnormalities associated with lipopolysaccharide-induced lecithin: cholesterol acyltransferase and lipase deficiency. J Biol Chem. 1989;264:10264-70.

33. Ettinger WH, Miller LD, Albers JJ, Smith TK, Parks JS. Lipopolysaccharide and tumor necrosis factor cause a fall in plasma concentration of lecithin: cholesterol acyltransferase in cynomolgus monkeys. J Lipid Res. 1990;31:1099-107.

34. Malmendier CL, Lontie JF, Sculier JP, Dubois DY. Modifications of plasma lipids, lipoproteins and apolipoproteins in advanced cancer patients treated with recombinant interleukin-2 and autologous lymphokine-activated killer cells. Atherosclerosis. 1988;73:173-80.

35. Rosenzweig IB, et al. Effects of interleukin-2 (IL-2) on human plasma lipid, lipoprotein, and C-reactive protein. Biotherapy. 1990;2:193-8.

36. Rosenzweig IB, Wiebe DA, Borden EC, Storer B, Shrago ES. Plasma lipoprotein changes in humans induced by beta-interferon. Atherosclerosis. 1987;67:261-7.

37. Spriggs DR, et al. Recombinant human tumor necrosis factor administered as a 24-hour intravenous infusion. A phase I and pharmacologic study. J Natl Cancer Inst. 1988;80:1039-44.

38. Levels JHM, Abraham PR, van Barreveld EP, Meijers JCM, van Deventer SJH. Distribution and kinetics of lipoprotein-bound lipoteichoic acid. Infect Immun. 2003;71:3280-4.
39. Voight BF, et al. Plasma HDL cholesterol and risk of myocardial infarction: a Mendelian randomisation study. Lancet (London, England). 2012;380:572-80.

40. Dodani $\mathrm{S}$, et al. Can dysfunctional HDL explain high coronary artery disease risk in South Asians? Int J Cardiol. 2008;129:125-32.

41. Van Lenten BJ, et al. Anti-inflammatory HDL becomes pro-inflammatory during the acute phase response: loss of protective effect of HDL against LDL oxidation in aortic wall cell cocultures. J Clin Invest. 1995;96:2758-67.

42. Dodani S, Dong L, Guirgis FW, Reddy ST. Carotid intima media thickness and low high-density lipoprotein (HDL) in South Asian immigrants: could dysfunctional HDL be the missing link? Arch Med Sci. 2014;10:870-9.

43. Navab M, Reddy ST, Van Lenten BJ, Anantharamaiah GM, Fogelman AM. The role of dysfunctional HDL in atherosclerosis. J Lipid Res. 2009;50(Suppl):S145-9.

44. Navab $\mathrm{M}$, et al. A cell-free assay for detecting HDL that is dysfunctional in preventing the formation of or inactivating oxidized phospholipids. J Lipid Res. 2001:42:1308-17.

45. Walley $\mathrm{KR}$, et al. PCSK9 is a critical regulator of the innate immune response and septic shock outcome. Sci Transl Med. 2014;6:258ra143.

46. Boyd JH, et al. Increased plasma PCSK9 levels are associated with reduced endotoxin clearance and the development of acute organ failures during sepsis. J Innate Immun. 2016;8:211-20.

47. Reddy ST, Navab M, Anantharamaiah GM, Fogelman AM. Apolipoprotein A-I mimetics. Curr Opin Lipidol. 2014;25:304-8.

48. Morin EE, Guo L, Schwendeman A, Li X-A. HDL in sepsis-risk factor and therapeutic approach. Front Pharmacol. 2015;6:244.

49. Lee $\mathrm{CR}$, Zeldin DC. Resolvin infectious inflammation by targeting the host response. N Engl J Med. 2015:373:2183-5.

50. Ouellette DR, Moscoso EE, Corrales JP, Peters M. Sepsis outcomes in patients receiving statins prior to hospitalization for sepsis: comparison of in-hospital mortality rates between patients who received atorvastatin and those who received simvastatin. Ann Intensive Care. 2015;5:9.

51. Wang HE, Griffin R, Shapiro NI, Howard G, Safford MM. Chronic Statin Use and Long-Term Rates of Sepsis: A Population-Based Cohort Study. J Intensive Care Med. 2016;31(6):386-96.

52. Thomas $\mathrm{G}$, et al. Statin therapy in critically-ill patients with severe sepsis: a review and meta-analysis of randomized clinical trials. Minerva Anestesiol. 2015;81:921-30.

53. Deshpande A, Pasupuleti $\vee$, Rothberg MB. Statin therapy and mortality from sepsis: a meta-analysis of randomized trials. Am J Med. 2015;128:410-7.e1.

\section{Submit your next manuscript to BioMed Central and we will help you at every step:}

- We accept pre-submission inquiries

- Our selector tool helps you to find the most relevant journal

- We provide round the clock customer support

- Convenient online submission

- Thorough peer review

- Inclusion in PubMed and all major indexing services

- Maximum visibility for your research

Submit your manuscript at www.biomedcentral.com/submit 